\title{
Prospects of mapping macromolecular structure and ionic gradients in hydrated biological specimens using Atom Probe Tomography
}

\author{
Daniel Perea $^{1}$ and Mark Wirth ${ }^{2}$ \\ ${ }^{1}$ EMSL at PNNL, United States, ${ }^{2}$ Pacific Northwest National Laboratory (PNNL), Richland, Washington, \\ United States
}

Within the field of materials science, the adage that structure determines properties is foundational to the field, while a similar adage underpins the field of structural biology where form follows function. This concept is easily exemplified with proteins, where their functions (from providing structural support, motility, transport, and enzymatic activity) is as varied as their unique amino acid sequence and complex physical 3-D structure. Currently, the application of individual or combinations of established analytical techniques such as cryo electron microscopy, nuclear magnetic resonance spectroscopy, mass spectrometry, and X-ray crystallography are used determine protein structure from ensembles of isolated proteins or protein crystals. However, the need to make measurements from ensembles of isolated proteins or crystals means that information is lost (for example, ionic gradients) with respect to the native aqueous environment. Here I will explore the question, can the analytical technique Atom Probe Tomography (APT) be applied to map macromolecular structure and ionic gradients with near atomic resolution within a hydrated environment? Recent work has established the ability of APT to map ionic gradients over nanoscale distances within a protein specimen embedded within a resin matrix[1]. More recently, established approaches for the preparation, handling, and transfer of cryogenically frozen hydrated specimens[2-5] provides a route for the site-specific targeting of hydrated biological samples for cryoAPT analysis, including regions containing proteins embedded in water ice. In this paper, we will discuss recent progress toward this end, which importantly includes the development of machine learning models aimed at mapping macromolecular structure from the 3-D point cloud composition maps. Additionally, we will discuss outstanding challenges that need to be addressed to realize the application of APT to directly map the structure of macromolecules such as proteins, and possible mitigation strategies.

\section{References}

[1] Perea, D.E., J. Liu, J. Bartrand, Q. Dicken, S.T. Thevuthasan, N.D. Browning, and J.E. Evans, Atom Probe Tomographic Mapping Directly Reveals the Atomic Distribution of Phosphorus in Resin Embedded Ferritin. Scientific Reports, 2016. 6: p. 22321.

[2] Perea, D.E., S.S.A. Gerstl, J. Chin, B. Hirschi, and J.E. Evans, An environmental transfer hub for multimodal atom probe tomography. Advanced Structural and Chemical Imaging, 2017. 3(12).

[3] Perea, D.E., D.K. Schreiber, J.V. Ryan, M.G. Wirth, L. Deng, X.N. Lu, J.C. Du, and J.D. Vienna, Tomographic mapping of the nanoscale water-filled pore structure in corroded borosilicate glass. Npj Materials Degradation, 2020. 4(1).

[4] Schreiber, D.K., D.E. Perea, J.V. Ryan, J.E. Evans, and J.D. Vienna, A method for site-specific and cryogenic specimen fabrication of liquid/solid interfaces for atom probe tomography. Ultramicroscopy, 2018. 194: p. 89-99.

[5] Zachman, M.J., N. de Jonge, R. Fischer, K.L. Jungjohann, and D.E. Perea, Cryogenic specimens for nanoscale characterization of solid-liquid interfaces. Mrs Bulletin, 2019. 44(12): p. 949-955. 